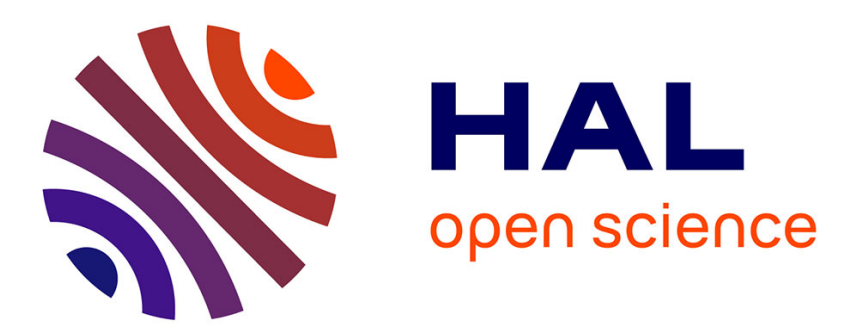

\title{
When Revision-Based Case Adaptation Meets Analogical Extrapolation
}

\author{
Jean Lieber, Emmanuel Nauer, Henri Prade
}

\section{To cite this version:}

Jean Lieber, Emmanuel Nauer, Henri Prade. When Revision-Based Case Adaptation Meets Analogical Extrapolation. 29th International Conference on Case-Based Reasoning (ICCBR 2021), Sep 2021, Salamanca (virtual), Spain. pp.156-170, 10.1007/978-3-030-86957-1_11 . hal-03483112

\section{HAL Id: hal-03483112 \\ https://hal.inria.fr/hal-03483112}

Submitted on 16 Dec 2021

HAL is a multi-disciplinary open access archive for the deposit and dissemination of scientific research documents, whether they are published or not. The documents may come from teaching and research institutions in France or abroad, or from public or private research centers.
L'archive ouverte pluridisciplinaire HAL, est destinée au dépôt et à la diffusion de documents scientifiques de niveau recherche, publiés ou non, émanant des établissements d'enseignement et de recherche français ou étrangers, des laboratoires publics ou privés. 


\title{
When Revision-Based Case Adaptation Meets Analogical Extrapolation
}

\author{
Jean Lieber ${ }^{1}$, Emmanuel Nauer ${ }^{1}$, and Henri Prade ${ }^{2}$ \\ ${ }^{1}$ Université de Lorraine, CNRS, Inria, LORIA, F-54000 Nancy, France \\ ${ }^{2}$ IRIT, CNRS, Université de Toulouse, France
}

\begin{abstract}
Case-based reasoning, where cases are described in terms of problem-solution pairs case $=(\mathrm{x}, \mathrm{y})$, amounts to propose a solution to a new problem on the basis of past experience made of stored cases. On the one hand, the building of the solution to a new problem may be viewed as a form of belief revision of the solution of a retrieved case (whose problem part is similar to the new problem) constrained by domain knowledge. On the other hand, an extrapolation mechanism based on analogical proportions has been proposed. It exploits triplets of cases $\left(\operatorname{case}^{a}, \operatorname{case}^{b}, \operatorname{case}^{c}\right)$ whose descriptions of problem parts $\mathrm{x}^{a}, \mathrm{x}^{b}, \mathrm{x}^{c}$ form an analogical proportion with the new problem $x^{\text {tgt }}$, in such a way that " $\mathrm{x}^{a}$ is to $\mathrm{x}^{b}$ as $\mathrm{x}^{c}$ is to $\mathrm{x}^{\mathrm{tgt}}$ ". Then, the analogical inference amounts to compute a solution $\mathrm{y}^{\text {tgt }}$ of $\mathrm{x}^{\text {tgt }}$ by solving (when possible) an equation expressing that " $\mathrm{y}^{a}$ is to $\mathrm{y}^{b}$ as $\mathrm{y}^{c}$ is to $\mathrm{y}^{\mathrm{tgt}}$ " (where $\mathrm{y}^{a}, \mathrm{y}^{b}$ and $\mathrm{y}^{c}$ are respectively the solution parts of $\operatorname{case}^{a}, \operatorname{case}^{b}$ and $\operatorname{case}^{c}$ ). The paper investigates how the belief revision view and analogical extrapolation relate. Besides that it constitutes an unexpected bridge between areas which ignore each other, it casts some light on the adaptation mechanism in case-based reasoning. The paper is illustrated by a running example.
\end{abstract}

Keywords: analogical inference, analogical proportion, belief revision, case-based reasoning, extrapolation

\section{Introduction}

Belief revision [2] and case-based reasoning [1] (CBR) are two areas of artificial intelligence that are usually thought of as quite distant and unrelated since the former takes place mainly in the setting of logic, while the latter deals with data and is similarity-based. Moreover, belief revision aims at reestablishing consistency after receiving a new piece of information that conflicts with the current state of belief. CBR has a quite different agenda since it is rather a matter of coping with missing information by taking advantage of similarity for completing a new problem with a plausible solution. However, note that the conclusions derived by belief revision or by CBR are only plausible in both approaches.

In spite of this apparent state of fact, there exists a belief revision-based view of CBR [14, 4]. Indeed, completing the new problem with a plausible solution may 
be viewed as adapting the solution of a retrieved case in order to be consistent with the specificities of the new case, which is a kind of revision. What is revised is clearly not the case base, but it is a copy of a case dealing with a similar problem. In other words, what is really revised is the allegation that the retrieved case can be applied (directly) to solve the target problem.

An extrapolation mechanism based on analogical proportions (which are statements of the form " $a$ is to $b$ as $c$ is to $d$ ") has been proposed for exploiting cases [16]. On the basis of 3 cases whose problem description parts are in analogical proportion with the new problem, one infers a solution for it from the solutions of the 3 cases. This means that an adaptation process takes place inside the inference. This is made possible by the fact that analogical proportions are a matter of both similarity and dissimilarity. Indeed when the problem parts of cases $a, b, c, d$ are described by vectors of features, the analogical proportion holds between the vectors if $a$ differs from $b$ in the same way $c$ differs from $d$ (and vice-versa) $[17,21]$. In that respect, a pair of cases may be viewed as encoding a kind of rule of adaptation in the sense of CBR [6].

The above points altogether suggest that there is a bridge between the belief revision-based view of CBR and analogical proportion-based extrapolation. This investigation is the topic of this paper. It is organized as follows. In Section 2, after offering a short refresher on propositional logic and introducing notations, two backgrounds are provided respectively on the analogical extrapolation of cases and on the belief revision view of CBR. This necessary setting of the problem addressed in this paper makes Section 2 rather long all the more as the running example is also introduced there. In order to bridge extrapolation and revision-based adaptation, Section 3 first reformulates adaptation by extrapolation as a single case adaptation. Then, a revision operator based on competence of case pairs is defined, which enables to establish that the adaptation by extrapolation can be equivalently obtained by a revision constrained by competent pairs expressing adaptation knowledge. Section 4 presents some related work and some concluding remarks, including a presentation of future work.

\section{Setting of the Problem and Running Example}

This section sets the notions related to the problem that this paper aims at solving, i.e., how adaptation by analogical extrapolation and revision-based adaptation can meet. For this purpose, the notions and notations used are presented, together with the introduction of a running example in the cooking domain.

\subsection{A quick refresher about propositional logic}

The formalism for representing cases and domain knowledge in this paper is propositional logic. Let $\mathcal{V}$ be a finite set of symbols, called variables. A formula is either a variable or an expression of one of the forms $T, \perp, \neg \varphi, \varphi_{1} \wedge \varphi_{2}$, $\varphi_{1} \vee \varphi_{2}, \varphi_{1} \rightarrow \varphi_{2}$, and $\varphi_{1} \leftrightarrow \varphi_{2}$ where $\varphi, \varphi_{1}$ and $\varphi_{2}$ are formulas. 
An interpretation $\mathcal{I}$ is a mapping from $\mathcal{V}$ to $\{0,1\}$ where 0 and 1 denote the Boolean values "false" and "true". The set of all interpretations is denoted by $\Omega$. An interpretation $\mathcal{I} \in \Omega$ is extended on every formulas as follows: $\mathcal{I}(T)=1$, $\mathcal{I}(\perp)=0, \mathcal{I}(\neg \varphi)=\operatorname{not} \mathcal{I}(\varphi), \mathcal{I}\left(\varphi_{1} \wedge \varphi_{2}\right)=\mathcal{I}\left(\varphi_{1}\right)$ and $\mathcal{I}\left(\varphi_{2}\right), \mathcal{I}\left(\varphi_{1} \vee \varphi_{2}\right)=$ $\mathcal{I}\left(\varphi_{1}\right)$ or $\mathcal{I}\left(\varphi_{2}\right), \mathcal{I}\left(\varphi_{1} \rightarrow \varphi_{2}\right)=\mathcal{I}\left(\neg \varphi_{1} \vee \varphi_{2}\right)$ and $\mathcal{I}\left(\varphi_{1} \leftrightarrow \varphi_{2}\right)=\mathcal{I}\left(\left(\varphi_{1} \rightarrow\right.\right.$ $\left.\left.\varphi_{2}\right) \wedge\left(\varphi_{2} \rightarrow \varphi_{1}\right)\right)$ where not, and and or are the classical Boolean operations. A model of a formula $\varphi$ is an interpretation $\mathcal{I}$ such that $\mathcal{I}(\varphi)=1$ and the set of models of $\varphi$ is denoted by $\mathcal{M}(\varphi)$. A formula $\varphi_{1}$ entails a formula $\varphi_{2}$, denoted by $\varphi_{1}=\varphi_{2}$, if $\mathcal{M}\left(\varphi_{1}\right) \subseteq \mathcal{M}\left(\varphi_{2}\right)$. The formulas $\varphi_{1}$ and $\varphi_{2}$ are equivalent, denoted by $\varphi_{1} \equiv \varphi_{2}$, if $\mathcal{M}\left(\varphi_{1}\right)=\mathcal{M}\left(\varphi_{2}\right)$. A formula $\varphi$ is consistent if $\mathcal{M}(\varphi) \neq \emptyset$.

Let $\Phi=\left\{\varphi_{1}, \varphi_{2}, \ldots, \varphi_{p}\right\}$ be a finite set of formulas. $\bigvee \Phi$ denotes $\varphi_{1} \vee \varphi_{2} \vee$ $\ldots \vee \varphi_{p} . \wedge \Phi$ denotes $\varphi_{1} \wedge \varphi_{2} \wedge \ldots \wedge \varphi_{p}$.

In the paper, for the sake of simplicity, the Boolean notations and the propositional notations are sometimes used together $(0$ and 1 instead of $T$ and $\perp$ as well as use of propositional connectives between Boolean values).

\subsection{Notions and notations related to CBR}

CBR aims at solving problems thanks to a case base, where a case is the representation of a problem-solving episode. Let $\mathcal{P}$ and $\mathcal{S}$ be the space of problems and solutions: a problem $\mathrm{x}$ (resp., a solution $\mathrm{y}$ ) is, by definition, an element of $\mathcal{P}$ (resp., of $\mathcal{S}$ ). A relation on $\mathcal{P} \times \mathcal{S}$ is assumed to exist that is read "has for solution" but is usually not completely known by the CBR system. A case is an ordered pair case $=(\mathrm{x}, \mathrm{y}) \in \mathcal{P} \times \mathcal{S}$ such that $\mathrm{x}$ has for solution $\mathrm{y}$. A source case case $^{s}=\left(\mathrm{x}^{s}, \mathrm{y}^{s}\right)$ is an element of the case base, which is denoted by CB. The current problem under solving is called the target problem, denoted by $\mathrm{x}^{\text {tgt }}$.

The classical CBR process model is based on the steps of retrieval and adaptation [24], also known as retrieve and reuse [1]. Other steps follow adaptation, but are not considered in this paper. Retrieval selects one or several source case(s) for the purpose of solving $\mathrm{x}^{\mathrm{tgt}}$. Adaptation aims at using the retrieved case(s) for proposing a solution $\mathrm{y}^{\text {tgt }}$ to $\mathrm{x}^{\text {tgt }}$. A single case adaptation is an adaptation of a sole retrieved case, otherwise, it is a multiple case adaptation.

The domain knowledge is a knowledge base DK that can be understood as a set of integrity constraints: a problem $\mathrm{x}$ (resp., a solution $\mathrm{y}$ or a case $(\mathrm{x}, \mathrm{y})$ ) that is inconsistent with DK is known to be not licit.

It is assumed in this paper that a clear separation of problems and solutions is made. This is not always true: for some applications of CBR, each case constitutes a whole, and a target problem is considered as an incomplete case, thus the problem-solution separation can be made at adaptation time. Since this paper is primarily concerned with adaptation, this assumption is not a big restriction. Therefore, in the propositional setting, $\mathcal{V}$ is partitioned into $\mathcal{V}_{\mathcal{P}}$ and $\mathcal{V}_{\mathcal{S}}$ and the variables occurring in a problem (resp., in a solution) are elements of $\mathcal{V}_{\mathcal{P}}$ (resp., of $\mathcal{V}_{\mathcal{S}}$ ). The next section illustrates this idea.

Furthermore, it is assumed that every source case $\left(\mathrm{x}^{s}, \mathrm{y}^{s}\right)$ and the target problem are fully described: given the domain knowledge, the truth value of each variable $a \in \mathcal{V}_{\mathcal{P}}$ is known for $\mathrm{x}^{s}$ and $\mathrm{x}^{\text {tgt }}$ (i.e., $\mathrm{DK} \wedge \mathrm{x}^{s} \models a$ or $\mathrm{DK} \wedge \mathrm{x}^{s} \models \neg a$ 
and the same for $\left.\mathrm{x}^{\text {tgt }}\right)$ and a similar constraint holds for $\mathrm{y}^{s}$. A case $\left(\mathrm{x}^{s}, \mathrm{y}^{s}\right)$ is represented in propositional logic by a single formula $\mathrm{x}^{s} \wedge \mathrm{y}^{s}$.

According to the classical knowledge model of CBR (see, e.g., [23]), the CBR knowledge base consists in four knowledge containers. Two of them have already been mentioned: the case base CB and the domain knowledge DK. The two others are the retrieval knowledge and the adaptation knowledge, used during the retrieval and adaptation steps.

A final remark can be made here about the notion of similarity in CBR: it is usually said that retrieval aims at finding cases similar to the target problem. Now, this notion of similarity should not necessarily be understood as an approximate equality: it may go beyond this. In fact, $\left(\mathrm{x}^{s}, \mathrm{y}^{s}\right)$ can be considered similar to $\mathrm{x}^{\text {tgt }}$ even if $\mathrm{x}^{s}$ and $\mathrm{x}^{\text {tgt }}$ descriptions are very different, considering that the adaptation of $\left(\mathrm{x}^{s}, \mathrm{y}^{s}\right)$ provides a plausible solution to $\mathrm{x}^{\text {tgt }}$. This is related to the adaptation-guided retrieval principle [25].

\subsection{Specification of the running example}

The example used throughout this paper is in the cooking domain. A recipe is represented simply by the type of ingredients it contains. Variables are food names representing recipe classes. For example, fruit represents the class of recipes having at least one fruit as ingredient. The domain knowledge is:

$$
\begin{aligned}
\mathrm{DK}=\{\text { pineapple } & \rightarrow \text { fruit }, & \text { cream } \rightarrow \text { sauce }, & \text { pesto } \rightarrow \text { sauce }, \\
\text { St Pierre } & \rightarrow \text { fish }, & \text { salmon } \rightarrow \text { fish }, & \neg(\text { fruit } \wedge \text { pesto })\}
\end{aligned}
$$

The last formula of DK states that a recipe must not have at the same time pesto (that contains garlic) and any fruit (fruit taken in the cooking sense of the term).

The source cases considered in this example are:

$$
\begin{aligned}
& \text { case }^{a}=\text { St Pierre } \wedge \text { cream } \wedge \text { vanilla } \wedge \text { curry } \wedge \text { Nothing else } \\
& \text { case }^{b}=\text { salmon } \wedge \text { cream } \wedge \text { vanilla } \wedge \text { pesto } \wedge \text { Nothing else } \\
& \text { case }^{s}=\text { St Pierre } \wedge \text { cream } \wedge \text { pineapple } \wedge \text { Nothing else }
\end{aligned}
$$

where, for a formula $\varphi, \varphi \wedge$ Nothing else denotes the formula $\varphi \wedge \Gamma$ where $\Gamma$ is the conjunction of the negative literals $\neg a$ such that $a \in \mathcal{V}$ and DK $\wedge \varphi \not \models a$. For example, case $^{s}$ contains the literal $\neg$ salmon.

The target problem is the request of a recipe with fish, pesto but no vanilla:

$$
\mathrm{x}^{\mathrm{tgt}}=\mathrm{fish} \wedge \text { pesto } \wedge \neg \text { vanilla }
$$

Thus, for the running example, $\mathcal{V}_{\mathcal{P}}=\{$ fish, pesto, vanilla $\}$ and $\mathcal{V}_{\mathcal{S}}=\mathcal{V} \backslash \mathcal{V}_{\mathcal{P}}$

\subsection{Analogical proportions and CBR}

Analogical proportions are statements of the form " $a$ is to $b$ as $c$ is $d$ ", denoted $a: b:: c: d$. Their origin dates back to Aristotle [3] (at least), and was inspired 
by a parallel with (geometric) numerical proportions, namely $\frac{a}{b}=\frac{c}{d}$; see [20]. In agreement with this parallel, they are supposed to obey the following postulates: Given a set of items $X$, analogical proportions form a quaternary relation supposed to obey the 3 following postulates (e.g., [13]): for $a, b, c, d \in X$,

1. $a: b:: a: b$ (reflexivity);

2. if $a: b:: c: d$ then $c: d:: a: b$ (symmetry);

3 . if $a: b:: c: d$ then $a: c:: b: d$ (central permutation).

The unique minimal Boolean model [22] obeying these 3 postulates is a quaternary propositional logic connective when $X$ is the Boolean set $\mathbb{B}=\{0,1\}[17]$ :

$$
a: b:: c: d=((a \wedge \neg b) \leftrightarrow(c \wedge \neg d)) \wedge((\neg a \wedge b) \leftrightarrow(\neg c \wedge d))
$$

It makes explicit that " $a$ differs from $b$ as $c$ differs from $d$ (and vice-versa)". It is easy to check that this formula is only valid for the 6 following valuations $0: 0:: 0: 0, \quad 1: 1:: 1: 1, \quad 0: 1:: 0: 1, \quad 1: 0:: 1: 0, \quad 0: 0:: 1: 1, \quad$ and $1: 1:: 0: 0$.

It can be seen that 1 and 0 play a symmetrical role, which makes the definition code-independent. This is formally expressed with the negation operator as: if $a: b:: c: d$ then $\neg a: \neg b:: \neg c: \neg d$. To deal with items, for instance cases, represented by vectors of Boolean values, the analogical proportion definition is extended componentwise from $X$ to $X^{n}$ :

$$
a: b:: c: d \quad \text { iff } \text { for all } i \in\{1, \ldots, n\}, a_{i}: b_{i}:: c_{i}: d_{i}
$$

This is the basis of an inference principle, first proposed in [18] for nominal values, that can be stated as follows:

$$
\frac{\forall i \in\{1, \ldots, n\}, \quad a_{i}: b_{i}:: c_{i}: d_{i} \text { holds }}{\forall j \in\{n+1, \ldots, m\}, \quad a_{j}: b_{j}:: c_{j}: d_{j} \text { holds }}
$$

As can be seen, knowledge from some components of source vectors is transferred to their remaining components, implicitly assuming that the values of the $n$ first components determine the values of the others.

This requires to find ? such that $a: b:: c:$ ? holds. The solution may not exist (e.g., for $0: 1:: 1:$ ?). It is solvable if and only if $a=b$ or $a=c$ in the Boolean case. Then, the unique solution is given by $?=c$ if $a=b$ and $?=b$ if $a=c$. Thus, we have the following property in the Boolean case

$$
a: a:: b: ? \text { if and only if } ?=b
$$

which is sometimes taken as a supplementary postulate and is not a consequence of the 3 postulates. This is the basis of the analogical extrapolation between cases proposed in [16].

Given a pair of vectors describing problems $\left(\mathrm{x}^{a}, \mathrm{x}^{b}\right)$, with $n$ components, their comparison yields a partition of the $n$ features in two subsets: the subset of features $\mathcal{E}_{\left(\mathrm{x}^{a}, \mathrm{x}^{b}\right)}$ for which the values of $\mathrm{x}^{a}$ and $\mathrm{x}^{b}$ are equal and the subset $\mathcal{D}_{\left(\mathrm{x}^{a}, \mathrm{x}^{b}\right)}$ for which they are different. Let us consider two pairs of vectors describing problems $\left(\mathrm{x}^{a}, \mathrm{x}^{b}\right)$ and $\left(\mathrm{x}^{c}, \mathrm{x}^{d}\right)$ such that $\mathcal{E}_{\left(\mathrm{x}^{a}, \mathrm{x}^{b}\right)}=\mathcal{E}_{\left(\mathrm{x}^{c}, \mathrm{x}^{d}\right)}$ and such that $\forall i \in$ $\mathcal{D}_{\left(\mathrm{x}^{a}, \mathrm{x}^{b}\right)}, \mathrm{x}^{a_{i}}=\mathrm{x}^{c_{i}}$ and $\mathrm{x}^{b_{i}}=\mathrm{x}^{d_{i}}$. Then $\mathrm{x}^{a}: \mathrm{x}^{b}:: \mathrm{x}^{c}: \mathrm{x}^{d}$ holds true, since 


\begin{tabular}{|c|c|c|c|c|c|c|c|c|c|c|}
\hline $\operatorname{case}^{a}$ & 1 & 0 & 1 & 1 & 0 & 0 & 4 & 8 & 7 & 4 \\
\hline $\operatorname{case}^{b}$ & 1 & 1 & 1 & 1 & 0 & 0 & 0 & 1 & 1 & 0 \\
\hline $\operatorname{case}^{s}$ & 1 & 0 & 0 & 1 & 1 & 1 & 1 & 0 & 1 & 0 \\
\hline$x^{\operatorname{tgt}}$ & 1 & 1 & 0 & $?$ & $?$ & $?$ & $?$ & $?$ & $?$ & $?$ \\
\hline case $_{\text {extrap }}^{\text {tgt }}$ & 1 & 1 & 0 & 1 & 1 & 1 & 0 & 1 & 1 & $?$ \\
\hline
\end{tabular}

Fig. 1. Analogical inference in the running example.

(i) for each $j \in \mathcal{E}_{\left(\mathrm{x}^{a}, \mathrm{x}^{b}\right)}=\mathcal{E}_{\left(\mathrm{x}^{c}, \mathrm{x}^{d}\right)},\left(\mathrm{x}^{a_{j}}, \mathrm{x}^{b_{j}}, \mathrm{x}^{c_{j}}, \mathrm{x}^{d_{j}}\right)$ is of the form $(u, u, v, v)$ or $(u, u, u, u)$ for some values $u$ and $v$ with $u \neq v$ and $u, v \in\{0,1\}$,

(ii) for each $k \in \mathcal{D}_{\left(\mathrm{x}^{a}, \mathrm{x}^{b}\right)}=\mathcal{D}_{\left(\mathrm{x}^{c}, \mathrm{x}^{d}\right)},\left(\mathrm{x}^{a_{k}}, \mathrm{x}^{b_{k}}, \mathrm{x}^{c_{k}}, \mathrm{x}^{d_{k}}\right)$ is of the form $(u, v, u, v)$.

The idea of looking at pairs of cases can be related to the reading of a pair $\left(\left(\mathrm{x}^{a}, \mathrm{y}^{a}\right),\left(\mathrm{x}^{b}, \mathrm{y}^{b}\right)\right)$ as a virtual rule expressing either that the change from $\mathrm{x}^{a}$ to $\mathrm{x}^{b}$ induces the change from $\mathrm{y}^{a}$ to $\mathrm{y}^{b}$, whatever the problem context (encoded by the features where $\mathrm{x}^{a}$ and $\mathrm{x}^{b}$ are equal), or that the change from $\mathrm{x}^{a}$ to $\mathrm{x}^{b}$ does not modify the solution (in case $\mathrm{y}^{a}=\mathrm{y}^{b}$ ).

However such virtual rules may have exception in the training set. Indeed there may exist $\left(\mathrm{x}^{a}, \mathrm{x}^{b}\right),\left(\mathrm{x}^{c}, \mathrm{x}^{d}\right)$ and $\left(\mathrm{x}^{a^{\prime}}, \mathrm{x}^{b^{\prime}}\right)$ such that

$-\mathrm{x}^{a}: \mathrm{x}^{b}:: \mathrm{x}^{c}: \mathrm{x}^{d}$ and $\mathrm{x}^{a^{\prime}}: \mathrm{x}^{b^{\prime}}:: \mathrm{x}^{c}: \mathrm{x}^{d}$ hold true. It means that $\mathcal{D}_{\left(\mathrm{x}^{a}, \mathrm{x}^{b}\right)}=\mathcal{D}_{\left(\mathrm{x}^{c}, \mathrm{x}^{d}\right)}=$ $\mathcal{D}_{\left(\mathrm{x}^{a^{\prime}}, \mathrm{x}^{b^{\prime}}\right)}$ and the changes from $\mathrm{x}^{a}$ to $\mathrm{x}^{b}$, from $\mathrm{x}^{c}$ to $\mathrm{x}^{d}$, from $\mathrm{x}^{a^{\prime}}$ to $\mathrm{x}^{b^{\prime}}$ are the same.

$-\mathrm{y}^{a}: \mathrm{y}^{b}:: \mathrm{y}^{c}: \mathrm{y}^{d}$ holds true.

$-\mathrm{y}^{a^{\prime}}: \mathrm{y}^{b^{\prime}}:: \mathrm{y}^{c^{\prime}}: \mathrm{y}^{d^{\prime}}$ does not hold for some feature $i$.

This may happen for instance when $\mathrm{y}^{a_{i}} \neq \mathrm{y}^{b_{i}}$ while $\mathrm{y}^{a_{i}^{\prime}}=\mathrm{y}^{b_{i}^{\prime}}$. In such a case, the two virtual rules associated to $\left(\left(\mathrm{x}^{a}, \mathrm{y}^{a_{i}}\right),\left(\left(\mathrm{x}^{b}, \mathrm{y}^{b_{i}}\right)\right)\right.$ and to $\left(\left(\mathrm{x}^{a^{\prime}}, \mathrm{y}^{a_{i}^{\prime}}\right),\left(\mathrm{x}^{b^{\prime}}, \mathrm{y}^{b_{i}^{\prime}}\right)\right)$ disagree. Then $\mathrm{y}^{a_{i}}=\mathrm{y}^{c_{i}}$ and $\mathrm{y}^{b_{i}}=\mathrm{y}^{d_{i}}$, but the solution of the equation $\mathrm{y}^{a_{i}^{\prime}}: \mathrm{y}^{b_{i}^{\prime}}:: \mathrm{y}^{c_{i}}: ? \mathrm{y}_{i}$ is $? \mathrm{y}_{i}=\mathrm{y}^{c_{i}} \neq \mathrm{y}^{d_{i}}$.

Thus the rate of exceptions of the virtual rule associated to a pair $\left(\left(\mathrm{x}^{a}, \mathrm{y}^{a_{i}}\right)\right.$, $\left.\left(\mathrm{x}^{b}, \mathrm{y}^{b_{i}}\right)\right)$ is an indication of the interest of the pair for analogical inference. This is called the competence of the case pair [15]. Note that each rule pertains to a particular feature used in the description of the solutions. Indeed it is not always so that there is a unique rule that computes the adaptation of $\mathrm{y}^{a}$ into $\mathrm{y}^{b}$ from the same problem context.

Let us apply analogical inference to the running example. It is easy to check that its propositional expression gives birth to the table on Figure 1. Considering the 3 cases $\left(\right.$ case $^{a}$, case $^{b}$, case $\left.^{s}\right)$, it appears that $\mathrm{x}^{a}: \mathrm{x}^{b}:: \mathrm{x}^{s}: \mathrm{x}^{\text {tgt }}$ holds true for the features fish, pesto and vanilla. Moreover, as an analogical equation $\mathrm{x}^{a}: \mathrm{x}^{b}:: \mathrm{x}^{s}: \mathrm{x}^{\mathrm{tgt}}$ can be solved for features sauce, fruit, pineapple, St Pierre, salmon, and cream. Thus, we obtain

$$
\begin{aligned}
\text { case }_{\text {extrap }}^{\text {tgt }} & =\mathrm{x}^{\text {tgt }} \wedge \mathrm{y}_{\text {extrap }}^{\text {tgt }} \\
\text { with } \mathrm{y}_{\text {extrap }}^{\text {tgt }} & \equiv \text { sauce } \wedge \text { fruit } \wedge \text { pineapple } \\
& \wedge \neg \text { St Pierre } \wedge \text { salmon } \wedge \text { cream }
\end{aligned}
$$


Some remarks are worth mentioning:

1. The analogical equation cannot be solved for feature curry, so nothing is inferred regarding its presence or absence from cases $\left(\operatorname{case}^{a}\right.$, case $\left.^{b}, \operatorname{case}^{s}\right)$. In a genuine example (where the case base would be richer), there may exist a triplet of cases enabling us to conclude on curry.

2. However, even if an exact resolution for curry does not exist, it would be possible to minimize an analogical dissimilarity measure $A D(a, b, c, d)$ for computing an "approximate" solution. $A D(a, b, c, d)$ is equal to the minimal number of flips for moving from $(a, b, c, d)$ to a 4-tuple corresponding to an analogical proportion: $A D(a, b, c, d)$ is maximal (and is equal to 2) if $(1,0,0,1)$ (or $(0,1,1,0))$ [17]. So here the approximate solution would be 0 (i.e., no curry) since $A D(1,0,0,0)=1$.

3 . In general, many triplets of cases can be applied to a given $x^{\text {tgt }}$. Remember that only the triplets built on the most competent pairs are used (when they lead to different conclusions a vote should take place).

4. As can be seen in the computation of case $\mathrm{t}^{\text {tgt }}$, a modification of case ${ }^{s}$ takes place: namely, in the context fish and $\neg$ vanilla with addition of pesto, case $^{s}$ is adapted by changing St Pierre into salmon.

5. In this example, some features are linked by implications. It should be noted that if for two mutually exclusive features $i$ and $j \mathrm{x}^{a_{i}}: \mathrm{x}^{b_{i}}:: \mathrm{x}^{c_{i}}: \mathrm{x}^{d_{i}}$ and $\mathrm{x}^{a_{j}}: \mathrm{x}^{b_{j}}:: \mathrm{x}^{c_{j}}: \mathrm{x}^{d_{j}}$ hold, this entail that it holds as well for a feature $k$ for which $i$ and $j$ are sub-classes, as can be seen on the example (for salmon, St Pierre, and fish). Thus, the analogical extrapolation makes no independence assumption between attributes.

6. One can observe that the roles of case ${ }^{b}$ and case ${ }^{s}$ could be exchanged, since analogical proportions are stable by central permutation.

\subsection{Belief revision and CBR}

This section summarizes the approach to adaptation based on belief revision as introduced in [14] and further developed in [4].

Belief revision. Let us consider an agent having a set of beliefs $\psi$ and that is confronted to another set of beliefs $\mu$ that are supposed to have priority over $\psi . \psi$ and $\mu$ are assumed in this paper to be represented in propositional logic. The question raised by belief revision is how the beliefs of the agent evolve by incorporation of $\mu$. When the new set of beliefs are not in contradiction with the old ones - i.e., $\psi \wedge \mu$ is consistent - then the revision gives simply this conjunction $\psi \wedge \mu$. Else, according to the minimal change principle of the socalled AGM theory (named after the authors of the paper [2]), belief revision consists in making a "minimal change" of $\psi$ into $\psi^{\prime}$ so that $\psi^{\prime} \wedge \mu$ is consistent, and then, the result of the revision is denoted by $\psi \dot{+} \mu=\psi^{\prime} \wedge \mu$. Now, the notion of change minimality is not uniquely defined and depends on how change is assessed, so many revision operators $\dot{+}$ exist. The AGM theory proposes a set of postulates that an operator $\dot{+}$ should respect. They have been formulated in propositional logic by [12] (for any formulas $\varphi, \psi, \varphi_{1}, \varphi_{2}, \psi_{1}, \psi_{2}$ and $\chi$ ): 
$(\dot{+} 1)$ If $\mu$ is consistent then $\psi \dot{+} \mu$ is consistent.

$(\dot{+} 2)$ If $\psi \wedge \mu$ is consistent then $\psi \dot{+} \mu \equiv \psi \wedge \mu$.

$(\dot{+} 3) \psi \dot{+} \mu \models \mu$.

$(\dot{+} 4)$ If $\psi_{1} \equiv \psi_{2}$ and $\mu_{1} \equiv \mu_{2}$ then $\psi_{1} \dot{+} \mu_{1} \equiv \psi_{2} \dot{+} \mu_{2}$.

$(\dot{+} 5)$ If $(\psi \dot{+} \mu) \wedge \chi$ are consistent then $\psi \dot{+}(\mu \wedge \chi) \models(\psi \dot{+} \mu) \wedge \chi$.

$(\dot{+} 6)(\psi \dot{+} \mu) \wedge \chi \models \psi \dot{+}(\mu \wedge \chi)$.

$(+1)$ states that the agent aims at having consistent beliefs (unless an inconsistent set of beliefs $\mu$ is accepted). ( +2$)$ is linked with the minimal change principle: if $\psi$ is consistent with $\mu$ then the minimal change $\psi \mapsto \psi^{\prime}$ is $\psi^{\prime}=\psi$ (i.e., no change). ( +3$)$ is related to the fact that $\mu$ has priority over $\psi$, i.e., the only belief changes are made on $\psi: \mu$ is unchanged. $(+4)$ states that revision respects the principle of independence to syntax (substituting a formula by an equivalent formula should not affect the result of the inference, up to equivalence). It can be shown that the conjunction of postulates $(\dot{+} 5)$ and $(\dot{+} 6)$ is equivalence to the following assertion: if $(\psi \dot{+} \mu) \wedge \chi$ is consistent then $\psi \dot{+}(\mu \wedge \chi) \equiv(\psi \dot{+} \mu) \wedge \chi$. In other words, if there is no need to further modify the beliefs after revision of $\psi$ by $\mu$ in order to incorporate $\chi$, then this additional modification is not performed and the new beliefs $\chi$ are simply added to the beliefs $\psi \dot{+} \mu$. This expresses the minimal change principle: when no further change is needed to restore consistency, then no such change is executed.

Despite these postulates, the set of belief revision operators is still wide, depending on the way change is assessed. In particular, it can be assessed thanks to a similarity measure between interpretations, giving birth to a family of revision operators presented below.

Similarity-based revision operators. A similarity measure on a set $S$ is defined in this paper as a function $\operatorname{sim}: S \times S \rightarrow[0,1] \operatorname{such}$ that $\operatorname{sim}(a, b)=1$ iff $a=b$ (for $a, b \in S$ ). A belief revision operator $\dot{+}^{\text {sim }}$ satisfying the AGM postulates can be defined for every similarity measure $\operatorname{sim}$ on $\Omega$, the set of interpretations:

$$
\begin{aligned}
\text { with } \operatorname{sim}^{*} & =\max \{\operatorname{sim}(\mathcal{I}, \mathcal{J}) \mid \mathcal{I} \in \mathcal{M}(\psi) \text { and } \mathcal{J} \in \mathcal{M}(\mu)\} \\
\mathcal{M}\left(\psi \dot{+}{ }^{\operatorname{sim}} \mu\right) & =\left\{\mathcal{J} \in \mathcal{M}(\mu) \mid \max _{\mathcal{I} \in \mathcal{M}(\psi)} \operatorname{sim}(\mathcal{I}, \mathcal{J})=\operatorname{sim}^{*}\right\}
\end{aligned}
$$

In other terms, the models of $\psi \dot{+}^{\text {sim }} \mu$ are the models of $\mu$ that are the most similar to models of $\psi$. This defines $\dot{+}^{\text {sim }}$ only up to logical equivalence, which is not a problem: any formula $\varrho$ such that $\varrho \equiv \psi \dot{+}^{\text {sim }} \mu$ constitutes the $\dot{+}^{\text {sim }}$ revision of $\psi$ by $\mu$ according to the principle of independence to syntax.

Given a distance function dist on $\Omega$, a similarity measure can be defined by $\operatorname{sim}(\mathcal{I}, \mathcal{J})=1 /(1+\operatorname{dist}(\mathcal{I}, \mathcal{J}))$ for $\mathcal{I}, \mathcal{J} \in \Omega$. In particular, let $H$ be the Hamming distance between interpretations: $H(\mathcal{I}, \mathcal{J})$ is the number of variables $a$ such that $\mathcal{I}(a) \neq \mathcal{J}(a)$. Let $\operatorname{sim}_{H}$ be the similarity measure associated with $H$. The revision operator $\dot{+}^{\operatorname{sim}_{H}}$ is the so-called Dalal revision operator [5], denoted by $\dot{+}_{\text {Dalal }}$ in the following. The Hamming distance weights every variable equally and considers variables independently: it can be seen as an edit distance on interpretations based on the "flip" edit operation that alters one variable at a time (turning 0 into 1 and conversely). For this reason, when there is no 
knowledge about how the change has to be measured, the Hamming distance being "neutral" is used for measuring this change. That is why, $\dot{+}_{\text {Dalal }}$ is used as a non informed operator, with an empty knowledge about change.

Revision-based adaptation is an approach to single case adaptation based on a revision operator $\dot{+}$. The intuition is that the modification of the retrieved case $\mathrm{x}^{s} \wedge \mathrm{y}^{s}$ in order to have a proposed solution to the target problem $\mathrm{x}^{\text {tgt }}$ is performed by $\dot{+}$. Both the retrieved case and the target problem are interpreted with the domain knowledge, hence the revision to be performed is that of $\mathrm{DK} \wedge \mathrm{x}^{s} \wedge \mathrm{y}^{s}$ by DK $\wedge \mathrm{x}^{\mathrm{tgt}}$. The result of this revision is a formula $\varrho$ entailing $\mathrm{x}^{\mathrm{tgt}}$ (according to postulate $(+3)$ ), thus $\varrho$ is equivalent to a formula $\mathrm{x}^{\text {tgt }} \wedge \mathrm{y}^{\text {tgt }}$ where all variables of $\mathrm{y}^{\text {tgt }}$ belong to $\mathcal{V}_{\mathcal{S}} \cdot \mathrm{y}^{\mathrm{tgt}}$ is the proposed solution of $\mathrm{x}^{\mathrm{tgt}}$. Formally, this can be written as follows:

$$
\left(\mathrm{DK} \wedge \mathrm{x}^{s} \wedge \mathrm{y}^{s}\right) \quad \dot{+}\left(\mathrm{DK} \wedge \mathrm{x}^{\mathrm{tgt}}\right) \quad \equiv \quad \mathrm{x}^{\mathrm{tgt}} \wedge \mathrm{y}^{\mathrm{tgt}}
$$

In order to apply revision-based adaptation, a revision operator has to be chosen. This choice is linked on how the change is assessed, that is, using the CBR terminology, the adaptation knowledge AK. So, when no adaptation knowledge is available $(A K=\emptyset)$, the Dalal revision operator is used.

It is noteworthy that the solution $\mathrm{y}^{\text {tgt }}$ provided by revision-based adaptation is necessarily consistent provided that $\mathrm{DK} \wedge \mathrm{x}^{\text {tgt }}$ is, but is not necessarily fully described: $\mathcal{M}\left(\mathrm{DK} \wedge \mathrm{x}^{\text {tgt }} \wedge \mathrm{y}^{\text {tgt }}\right)$ may contain several interpretations. In such a situation, this means that the revision-based adaptation asserts that there exists a plausible solution $\mathrm{y}$ to $\mathrm{x}^{\text {tgt }}$ that verifies $\mathrm{y} \models \mathrm{y}^{\text {tgt }}$. In the extreme situation, $\mathrm{y}^{\text {tgt }} \equiv \mathrm{T}$, meaning that the revision-based adaptation gives no information on a potential solution of $\mathrm{x}^{\text {tgt }}$.

The running example can be solved using revision-based adaptation. The retrieved case is case ${ }^{s}$ and the target problem is $x^{\text {tgt }}$, defined by (3) and (4). Let us consider this adaptation using $\dot{+}_{\text {Dalal }}$. It can be shown that the result $\mathrm{y}^{\text {tgt }}$ of this revision is

$$
\begin{aligned}
\mathrm{y}_{\text {Dalal }}^{\text {tgt }} & \equiv \text { St Pierre } \wedge \text { cream } \wedge \text { sauce } \\
& \wedge \neg \text { fruit } \wedge \neg \text { pineapple } \wedge \neg \text { salmon } \wedge \neg \text { curry }
\end{aligned}
$$

So, this adaptation consists in removing from case $e^{s}=\mathrm{x}^{s} \wedge \mathrm{y}^{s}$ the fruits because their presence would be inconsistent with $\mathrm{DK} \wedge \mathrm{x}^{\mathrm{tgt}}$.

\section{Bridging Extrapolation and Revision-Based Adaptation}

The two approaches to case adaptation presented above - the one based on analogical extrapolation and the one based on belief revision - appear to be quite different: the first one is a multiple case adaptation approach whereas the second one is a single case one (relying respectively on the retrieval of source cases by triplets and by singletons). Nevertheless, the goal of this section is to show how they can meet. First, the approach based on extrapolation is reformulated as a 
single case adaptation. Second, a revision operator based on source case pairs is defined and it is shown how, under some circumstances, the two approaches to adaptation coincide. This makes it possible to define an approach to adaptation based on both extrapolation and revision that takes into account, on the one hand, the case base and the case pair competence, and, on the other hand, the domain knowledge.

\subsection{Reformulating adaptation by extrapolation as a single case adaptation}

In the above presentation of analogical extrapolation, it has been considered that a triplet of cases $\left(\operatorname{case}^{a}\right.$, case $^{b}$, case $\left.^{c}\right)$ is retrieved and then reused in order to solve $\mathrm{x}^{\text {tgt }}$. Now, this can be reformulated in a new way by considering that only case $^{c}$ is retrieved, and the other ones, case ${ }^{a}$ and case ${ }^{b}$, are selected during the adaptation process itself. This "symmetry breaking" has two advantages. First, it can be used for the purpose of an efficient implementation (this issue can be related to the issue of implementing extrapolation algorithms presented in [16]). Second, it makes it possible to match the two approaches to adaptation; for this reason, the retrieved case case ${ }^{c}$ is renamed case $\mathrm{c}^{s}=\left(\mathrm{x}^{s}, \mathrm{y}^{s}\right)$, to better match the notations of single case adaptation.

Therefore, the reformulation of analogical extrapolation as a single case adaptation is as follows:

Input: the case to be adapted $\left(\mathrm{x}^{s}, \mathrm{y}^{s}\right)$, the target problem $\mathrm{x}^{\text {tgt }}$, the case base $\mathrm{CB}$, the preference relation between pairs of cases

Output: a set of proposed solutions $\mathrm{Y}$ to $\mathrm{x}^{\text {tgt }}$

1. Let CandidateCasePairs be the set of $\left(\right.$ case $^{a}$, case $\left.^{b}\right) \in \mathrm{CB} \times \mathrm{CB}$ such that $\mathrm{x}^{a}: \mathrm{x}^{b}:: \mathrm{x}^{s}: \mathrm{x}^{\text {tgt }}$ holds and the analogical equation $\mathrm{y}^{a}: \mathrm{y}^{b}:: \mathrm{y}^{s}: ? \mathrm{y}$ is solvable.

2. Let BestCandidateCasePairs be the set of most competent case pairs among CandidateCasePairs.

3. Let $\mathrm{Y}=\left\{\begin{array}{l|l}\mathrm{y} & \begin{array}{l}\mathrm{y} \text { is the solution of } \mathrm{y}^{a}: \mathrm{y}^{b}:: \mathrm{y}^{s}: ? \mathrm{y} \\ \text { for }\left(\text { case }^{a}, \text { case }^{b}\right) \in \text { BestCandidateCasePairs }\end{array}\end{array}\right\}$.

4. $\mathrm{Y}$ is returned as a set of candidate solutions to $\mathrm{x}^{\mathrm{tgt}}$.

\subsection{A revision operator based on competence of case pairs}

A similarity measure $\operatorname{sim}_{\text {comp }}$ on $\Omega$ that is based on pairs of cases and case pair competence can be defined under some assumptions, hence the revision operator

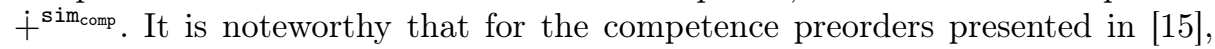
these assumptions hold.

The first assumptions is that the competence preorder between pairs of cases can be defined thanks to a competence level, i.e., a function compLvl that maps a pair of source cases to a value in $[0,1]$ such that $\left(\right.$ case $^{1}$, case $\left.^{2}\right)$ is deemed to be strictly more competent than $\left(\operatorname{case}^{3}, \operatorname{case}^{4}\right)$ iff $\operatorname{compLvl}\left(\operatorname{case}^{1}, \operatorname{case}^{2}\right)>$ compLvl (case ${ }^{3}$, case $\left.^{4}\right)$. 
The second assumption just translates the fact that two pairs of cases being in analogy have the same competence, hence the same competence level:

$$
\begin{aligned}
& \text { if } \mathrm{x}^{a}: \mathrm{x}^{b}:: \mathrm{x}^{c}: \mathrm{x}^{d} \text { and } \mathrm{y}^{a}: \mathrm{y}^{b}:: \mathrm{y}^{c}: \mathrm{y}^{d} \\
& \text { then compLvl }\left(\operatorname{case}^{a}, \operatorname{case}^{b}\right)=\operatorname{compLv}\left(\operatorname{case}^{c}, \text { case }^{d}\right)
\end{aligned}
$$

The third assumption is that the maximum level of competence is 1 and is reached only by pairs (case ${ }^{a}$, case $^{a}$ ), for case ${ }^{a} \in \mathrm{CB}$. This third assumption can be justified by the fact that if $\mathrm{x}^{a}: \mathrm{x}^{a}:: \mathrm{x}^{s}: \mathrm{x}^{\text {tgt }}$ then $\mathrm{x}^{s}=\mathrm{x}^{\text {tgt }}$ (according to (5)) and thus that the analogical equation $\mathrm{y}^{a}: \mathrm{y}^{a}:: \mathrm{y}^{s}: ? \mathrm{y}$ has exactly one solution ?y $=\mathrm{y}^{s}$ that solves $\mathrm{x}^{\text {tgt }}=\mathrm{x}^{s}$.

The fourth assumption is that the level of competence is minimal for $\left(\operatorname{case}^{a}\right.$, case $\left.^{b}\right)$ iff this case pair is in analogy with no case pair $\left(\right.$ case $^{c}$, case $\left.^{d}\right) \in$ $\mathrm{CB}^{2}$ (which involves that $\left\{\right.$ case $^{a}$, case $\left.^{b}\right\} \nsubseteq \mathrm{CB}$ ).

A one-to-one correspondence caseOf between $\Omega$ and the set of fully described cases can be defined, for $\mathcal{I} \in \Omega$, by $\operatorname{caseOf}(\mathcal{I})=(\mathrm{x}, \mathrm{y})$ is such that $\mathcal{M}(\mathrm{x} \wedge \mathrm{y})=$ $\{\mathcal{I}\}$. A similarity measure $\operatorname{sim}_{\text {comp }}$ can be defined using this correspondence and the competence level function:

$$
\operatorname{sim}_{\text {comp }}(\mathcal{I}, \mathcal{J})=\operatorname{compLvl}(\operatorname{case0f}(\mathcal{I}), \operatorname{case0f}(\mathcal{J})) \quad(\text { for } \mathcal{I}, \mathcal{J} \in \Omega)
$$

The meeting between the two approaches of adaptation is expressed by the following result (given a source case $\left(\mathrm{x}^{s}, \mathrm{y}^{s}\right)$ and a target problem $\mathrm{x}^{\mathrm{tgt}}$ ):

if $\mathrm{y}^{\text {tgt }}$ is the result of revision-based adaptation with $\dot{+}=\dot{+}^{\text {sim }_{\text {comp }}}$, the domain knowledge is empty $(\mathrm{DK}=T)$,

$\mathrm{Y}$ is the set of solutions obtained by analogical extrapolation and $\mathrm{Y} \neq \emptyset$

then $\mathrm{y}^{\mathrm{tgt}} \equiv \bigvee \mathrm{Y}$

In other terms, in absence of any domain knowledge, revision-based adaptation using the revision operator $\dot{+}^{\text {sim comp }}$ based on case pair competence gives the same result as analogical extrapolation, unless this latter gives an empty set of solution. Furthermore, it can be shown that if this set is empty, then $\mathrm{y}^{\mathrm{tgt}} \equiv T$, i.e., in this case, revision-based adaptation gives no information on the solution.

The proof of (11) mainly consists in applying the definitions: the similarity

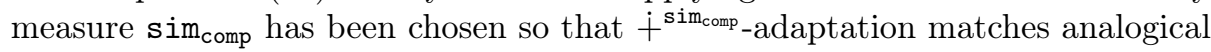
extrapolation when DK is empty.

\subsection{An approach to adaptation based on extrapolation and revision}

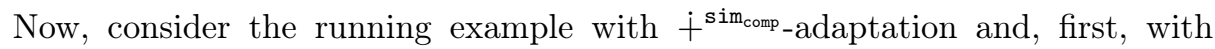
$\mathrm{DK}=\emptyset$. Because of the variable curry, analogical extrapolation gives no solution $^{1}(\mathrm{Y}=\emptyset)$ and in this situation, $\mathrm{y}^{\mathrm{tgt}} \equiv \top$ (no information on the solution

\footnotetext{
${ }^{1}$ More precisely, the analogical equation $\mathrm{y}^{a}: \mathrm{y}^{b}:: \mathrm{y}^{s}: \mathrm{y}$ has no solution: it is solvable feature by feature on every feature except curry, so a solution can be proposed for every other features.
} 
is proposed). If the curry variable is discarded from $\mathcal{V}$ then, according to (11), the proposed solution verifies:

$$
\mathrm{y}^{\mathrm{tgt}}=\mathrm{y}_{+}^{\mathrm{tgt}}{ }^{\text {sim }} \text { tomp }_{\text {-adaptation without curry }} \equiv \mathrm{y}_{\text {extrap }}^{\mathrm{tgt}}
$$

Now, in order to design an approach that considers all the variables, even the ones such as curry that does not match exactly extrapolation, a similarity measure $\operatorname{sim}_{\mathrm{E} \& \mathrm{D}}$ can be defined that combines $\operatorname{sim}_{\mathrm{comp}}$ and $\operatorname{sim}_{H}$ (for a given $\alpha, 0<\alpha<1$, and $\mathcal{I}, \mathcal{J} \in \Omega$ ):

$$
\operatorname{sim}_{\mathrm{E} \& \mathrm{D}}(\mathcal{I}, \mathcal{J})=(1-\alpha) \operatorname{sim}_{\mathrm{comp}}(\mathcal{I}, \mathcal{J})+\alpha \operatorname{sim}_{H}(\mathcal{I}, \mathcal{J})
$$

hence the revision operator $\dot{+}^{\text {simE\&D }}$ (E\&D for "extrapolation and Dalal"). Then, for a small enough $\alpha, \dot{+}^{\mathrm{sim}_{\mathrm{E} \& \mathrm{D}}}$-adaptation consists in making a minimal number of variable flips on case $^{s}$ to make extrapolation possible and then in applying it (in fact, it is sufficient that $\alpha<(2|\mathcal{V}|)^{-1}$. ). In particular, if analogical extrapolation applies (on every variables) then no flip is necessary and $\dot{+}^{\text {SimE\&D }}$-adaptation

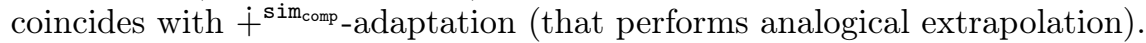

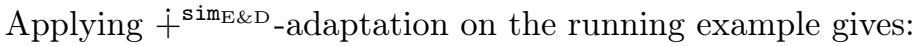

$$
\begin{aligned}
\mathrm{y}_{\mathrm{E} \& \mathrm{D}}^{\mathrm{tgt}} & \equiv \text { sauce } \wedge \text { fruit } \wedge \text { pineapple } \\
& \wedge \neg \text { St Pierre } \wedge \text { salmon } \wedge \text { cream } \wedge \neg \text { curry }
\end{aligned}
$$

The analogical equation $1: 0:: 0: ? \mathrm{y}_{\text {curry }}$ for finding the value of the feature curry of $\mathrm{y}^{\text {tgt }}$ by extrapolation of (case ${ }^{a}$, case $^{b}$, case $\left.^{s}\right)$ has no solution, so a flip of this feature for case ${ }^{s}$ gives case ${ }^{s \prime}$ and the triplet $\left(\operatorname{case}^{a}\right.$, case $^{b}$, case $\left.^{s \prime}\right)$ can be used by extrapolation to solve $\mathrm{x}^{\mathrm{tgt}}$ in $\mathrm{y}^{\mathrm{tgt}}=\mathrm{y}_{\mathrm{E} \& \mathrm{D}}^{\mathrm{tgt}}$, since $1: 0:: 1: \mathrm{y}_{\text {curry }}$ has a unique solution $? \mathrm{y}_{\text {curry }}=0$ (hence $\neg$ curry in the proposed solution of $\mathrm{x}^{\mathrm{tgt}}$ ).

Now, this adaptation has not taken into account the domain knowledge and, in fact, DK $\wedge \mathrm{x}^{\mathrm{tgt}} \wedge \mathrm{y}_{\mathrm{E} \& \mathrm{D}}^{\mathrm{tgt}}$ is inconsistent, with the DK of the running example (because of the pesto-fruit conflict). Therefore, the proposed approach to adaptation consists in doing a $\dot{+}^{\text {sim }_{E} \& D}$-adaptation taking into account DK as in (8). With the running example, this gives:

$$
\begin{aligned}
\mathrm{y}_{\mathrm{E} \& \mathrm{D} \text { w } / \mathrm{DK}}^{\mathrm{tgt}} & \equiv \text { sauce } \wedge \neg \text { fruit } \wedge \neg \text { pineapple } \\
& \wedge \neg \text { St Pierre } \wedge \text { salmon } \wedge \text { cream } \wedge \neg \text { curry }
\end{aligned}
$$

which consists in removing fruits from $\mathrm{y}_{\mathrm{E} \& \mathrm{D}}^{\mathrm{tgt}}$.

\subsection{Synthesis}

Figure 2 describes the cases and the target problem presented above, as well as the proposed cases case ${ }^{s}=\mathrm{x}^{\text {tgt }} \wedge \mathrm{y}^{\text {tgt }}$ after the different adaptation processes.

This example illustrates how the strengths of two approaches to adaptation can be combined: 


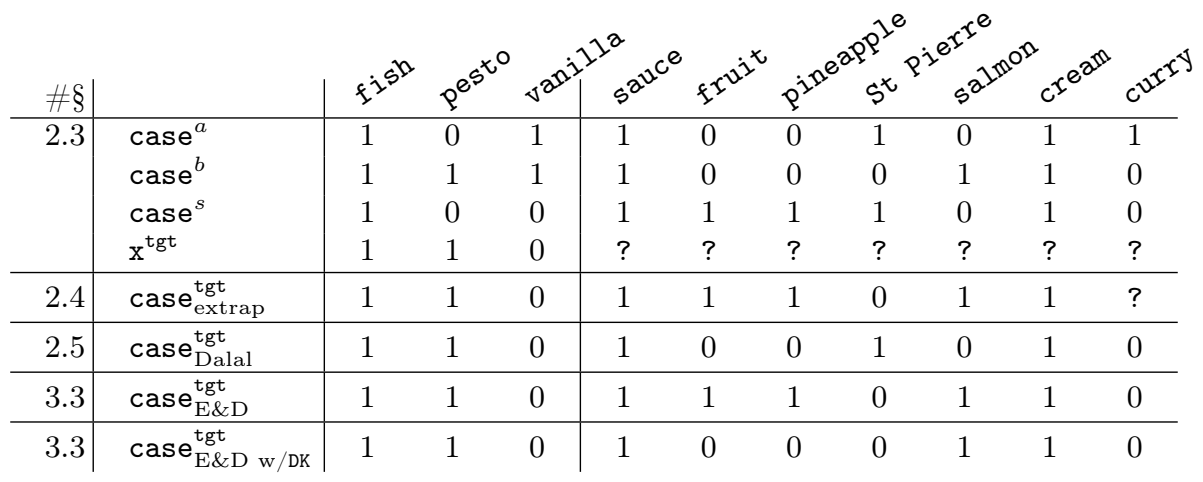

Fig. 2. The running example used throughout the paper: problem setting and outcomes case $^{\text {tgt }}=\mathrm{x}^{\text {tgt }} \wedge \mathrm{y}^{\text {tgt }}$ of adaptation processes presented in the paper. The column $\# \S$ indicates the number of the relevant section.

- Analogical extrapolation's strength is to exploit the variations within the case base (variations that can be seen as specific adaptation rules): the variation "Saint-Pierre to salmon" from case ${ }^{a}$ to $\operatorname{case}^{b}$ is applied to case ${ }^{s}$ in a similar context.

- $\dot{+}_{\text {Dalal-adaptation's strength is to take into account the domain knowledge }}$ in order to adjust the retrieved case to propose a solution to the target problem: the pesto being incompatible with fruits, pineaple is removed from the recipe. ${ }^{2}$

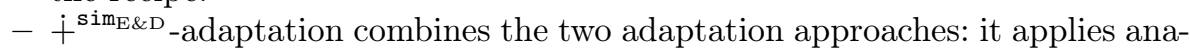
logical extrapolation on each feature for which it is both possible and consistent with the domain knowledge, and adjust the other features in the $\dot{+}_{\text {Dalal-adaptation way. }}$

\section{Related work and final remarks}

This paper has considered two very different approaches to case adaptationanalogical extrapolation and revision-based adaptation - and has investigated the issue of how they can meet. It has been shown that, under some circumstances (propositional setting, no domain knowledge, etc.), they coincide (cf. (11)) and that the approach can be extended when domain knowledge is added and/or when analogical proportions holds only for some solution features. The idea is that the case pairs and their competences - used in analogical extrapolationcan be used for "re-shaping the adaptation space" by making more similar the source case and the target problem, and that this similarity is used by the revision operator.

Related work. There is a rich literature on belief revision following the seminal work of Alchourrón, Gärdenfors and Makinson [2], and its expression in a propositional logic setting [12]. However, the idea of applying belief revision to CBR,

\footnotetext{
${ }^{2}$ It is noteworthy that $\dot{+}_{\text {Dalal-adaptation may do more than removing positive facts }}$ as it can substitute a class by a sibling class in the taxonomy (see, e.g., [14]).
} 
as restated in the preliminaries of this paper, can be found only in few works; see [4] in particular.

The idea of an analogical inference pattern based on analogical proportions dates back to [18]. Its application to CBR is suggested in [21], and more systematically investigated in [16].

There has been no work bridging analogical extrapolation and belief revision until now. Keeping in mind that belief revision and nonmonotonic logic are two sides of the same coin in some sense [10], we may however mention a discussion [19] contrasting nonmonotonic reasoning and analogical reasoning, but also providing pathways between them.

Future work. This paper has shown how two models of adaptation can meet, but the question remain of the practical usefulness of this meeting, beyond the running example. For this purpose, a first line of future work is to conduct an experiment comparing the different approaches presented there.

The revision operators $\dot{+}^{\text {sim }{ }_{\text {comp }}}$ and $\dot{+}^{\text {simE\&D }}$ may change with the addition of a new case in the case base, e.g., the case $\left(\mathrm{x}^{\mathrm{tgt}}, \mathrm{y}^{\mathrm{tgt}}\right)$ when this new case is validated. Indeed, the competence level of case pairs is computed on the basis of $\mathrm{CB}$. The evolution of revision operators over time is an issue related to iterated revision (see, e.g., [7]), so, it would be interesting to study $\dot{+}^{\text {sim }_{\text {comp }}}$ and $\dot{+}{ }^{\text {sime\&D }}$ at the light of the postulates of iterated revision postulates.

Lastly, it is known that belief revision can be encoded in possibilistic logic [9], since belief revision relies at the semantic level on epistemic entrenchment relations [11], which are nothing but qualitative necessity relations in the sense of possibility theory [8]. How to process the different kinds of revision / adaptation considered in this paper in the possibilistic setting is another topic for future research.

\section{References}

1. Aamodt, A., Plaza, E.: Case-based Reasoning: Foundational Issues, Methodological Variations, and System Approaches. AI Communications 7(1) (1994) 39-59

2. Alchourrón, C.E., Gärdenfors, P., Makinson, D.: On the logic of theory change: partial meet functions for contraction and revision. J. Symbolic Logic 50 (1985) $510-530$

3. Aristotle: Nicomachean Ethics. Univ. of Chicago Press (2011) Trans. by R. C. Bartlett and S. D. Collins.

4. Cojan, J., Lieber, J.: Applying belief revision to case-based reasoning. In Prade, H., Richard, G., eds.: Computational Approaches to Analogical Reasoning: Current Trends. Volume 548 of Studies in Computational Intelligence. Springer (2014) 133161

5. Dalal, M.: Investigations into a theory of knowledge base revision: Preliminary report. In: Proceedings of the Seventh National Conference on Artificial Intelligence (AAAI). (1988) 475-479

6. d'Aquin, M., Badra, F., Lafrogne, S., Lieber, J., Napoli, A., Szathmary, L.: Case base mining for adaptation knowledge acquisition. In Veloso, M.M., ed.: IJCAI 
2007, Proc. of the 20th Int. Joint Conf. on Artificial Intelligence, Hyderabad, Jan. 6-12. (2007) 750-755

7. Darwiche, A., Pearl, J.: On the logic of iterated belief revision. Artificial intelligence 89(1-2) (1997) 1-29

8. Dubois, D., Prade, H.: Epistemic entrenchment and possibilistic logic. Artif. Intell. 50(2) (1991) 223-239

9. Dubois, D., Prade, H.: Possibilistic logic - An overview. In Siekmann, J.H., ed.: Computational Logic. Volume 9 of Handbook of the History of Logic. Elsevier (2014) 283-342

10. Gärdenfors, P.: Belief revision and nonmonotonic logic: Two sides of the same coin? In: Proc. 9th Europ. Conf. on Artificial Intelligence (ECAI'90), Stockholm. (1990) 768-773

11. Gärdenfors, P., Makinson, D.: Revisions of knowledge systems using epistemic entrenchment. In Vardi, M.Y., ed.: Proc. 2nd Conf. on Theoretical Aspects of Reasoning about Knowledge, Pacific Grove, Morgan Kaufmann (1988) 83-95

12. Katsuno, H., Mendelzon, A.: Propositional knowledge base revision and minimal change. Artificial Intelligence 52(3) (1991) 263-294

13. Lepage, Y.: Analogy and formal languages. Electr. Notes Theor. Comput. Sci. 53 (2001)

14. Lieber, J.: Application of the revision theory to adaptation in case-based reasoning: The conservative adaptation. In Weber, R., Richter, M.M., eds.: Case-Based Reasoning Research and Development, 7th Int. Conf. on Case-Based Reasoning, ICCBR 2007, Belfast, Proc. Volume 4626 of LNCS., Springer (2007) 239-253

15. Lieber, J., Nauer, E., Prade, H.: Improving analogical extrapolation using case pair competence. In: Case-Based Reasoning Research and Development, 27th International Conference (ICCBR-2019), Otzenhausen, France (September 2019)

16. Lieber, J., Nauer, E., Prade, H., Richard, G.: Making the best of cases by approximation, interpolation and extrapolation. In: ICCBR 2018 - 26th International Conference on Case-Based Reasoning, Stockholm, Sweden, Springer (July 2018)

17. Miclet, L., Prade, H.: Handling analogical proportions in classical logic and fuzzy logics settings. In: Proc. 10th Eur. Conf. on Symbolic and Quantitative Approaches to Reasoning with Uncertainty (ECSQARU'09), Springer, LNCS 5590 (2009) 638650

18. Pirrelli, V., Yvon, F.: Analogy in the lexicon: a probe into analogy-based machine learning of language. In: Proc. 6th Int. Symp. on Human Communication. (Santiago de Cuba, 6 p.,1999)

19. Prade, H., Richard, G.: Cataloguing/analogizing: A nonmonotonic view. Int. J. Intell. Syst. 26(12) (2011) 1176-1195

20. Prade, H., Richard, G.: From analogical proportion to logical proportions. Logica Universalis 7(4) (2013) 441-505

21. Prade, H., Richard, G.: Analogical proportions and analogical reasoning - An introduction. In Aha, D.W., Lieber, J., eds.: Proc. 25th Int. Conf. on Case-Based Reasoning Research and Development (ICCBR'17), Trondheim, Norway, June 2628. Volume 10339 of LNAI., Springer (2017) 16-32

22. Prade, H., Richard, G.: Analogical proportions: From equality to inequality. Int. J. of Approximate Reasoning 101 (2018) $234-254$

23. Richter, M.M., Weber, R.O.: Case-based reasoning, a textbook. Springer (2013)

24. Riesbeck, C.K., Schank, R.C.: Inside Case-Based Reasoning. Lawrence Erlbaum Associates, Inc., Hillsdale, New Jersey (1989) Available on line.

25. Smyth, B., Keane, M.T.: Using adaptation knowledge to retrieve and adapt design cases. Knowledge-Based Systems 9(2) (1996) 127-135 